\title{
Comparison of non-convex cost functionals for the consideration of phase differences in phaseless near-field far-field transformations of measured antenna fields
}

\author{
Josef Knapp, Alexander Paulus, Carlos Lopez, and Thomas F. Eibert \\ Chair of High-Frequency Engineering, Technical University of Munich, Arcisstr. 21, 80333 Munich, Germany \\ Correspondence to: Josef Knapp (josef.knapp@tum.de)
}

Received: 19 December 2016 - Revised: 10 March 2017 - Accepted: 13 March 2017 - Published: 21 September 2017

\begin{abstract}
This work introduces two methods which extend the non-convex minimization problem arising in phaseless (NF) far-field (FF) transformations. With the new extensions, knowledge about phase differences between measurement points can be incorporated into the minimization problem. The additional information helps to avoid stationary points of the minimization cost functional which would otherwise compromise the result of the near-field far-field transformation. The methods are incorporated into the Fast Irregular Antenna Field Transformation Algorithm (FIAFTA), analyzed and compared. Their effectiveness is shown by transforming synthetic near-field data sets with partial knowledge of phase differences to the far-field.
\end{abstract}

\section{Introduction}

With the rapid development in communication technology, also the demands for antennas and antenna measurement technologies increase. In antenna measurements, one of the main interests is to determine the antenna radiation pattern in the far-field (FF) of the antenna under test (AUT). If straightforward measurements in the AUT FF are not feasible, for example when the FF distance exceeds the measurement chamber dimensions, then the measurements can be obtained in the near-field (NF) of the AUT and afterwards the AUT FF pattern can be determined by NF to FF transformation (NFFFT). In general, magnitude and phase information are required in the NF measurements in order to determine the FF. However, phase measurements become complicated in the very high frequency regime or might also be omitted when utilizing cheap scalar measurement equipment. Phase- less NFFFTs, which require only magnitude data, are needed for the development of leading-edge antenna technologies. The phaseless NFFFT problem is directly related to the more general problem of phase retrieval, which has numerous applications in optics, radiology, and various physical disciplines (Waldspurger et al., 2015; Fienup, 1982; Wu et al., 2005). Since the measurements in phase retrieval scenarios (i.e. the radiation from an antenna for example) arise in a well defined physical environment, there exists a relationship between the magnitude of the measurements and its phase. Due to the non linear nature of this relationship, the phase can not easily be retrieved from magnitude only measurements.

First successful attempts in retrieving the phase of magnitude only data are found in Gerchberg (1972). The initial algorithm has been further developed throughout the years (for example in Fienup, 1982) and the topic is still under heavy research. In the last decade, one has often tried to retrieve the phase via non-convex minimization (Netrapalli et al., 2013; Candes et al., 2015; Zhang and Liang, 2016) or by a convex relaxation of the non-convex formulation (Waldspurger et al., 2015; Yurtsever et al., 2015; Candes et al., 2013; Bauschke et al., 2002). Because of their high numerical complexity, convex relaxations are suitable for small and medium sized problems only. Non-convex minimizations suffer from stationary points of the cost functional. Convergence guarantees have been established for the non-convex minimization in Candes et al. (2015); Zhang and Liang (2016) and Netrapalli et al. (2013). However, the convergence criteria hold only, if enough measurements can be obtained, which follow certain probability distributions. It remains unclear, how such measurements can be obtained in a NF antenna measurement scenario. Interferometric methods have been used to obtain phase differences from magnitude only measure- 
ments in Costanzo et al. (2005). There, the measured phase differences have been set up along a chain from one element to the other, thus being very inflexible in the measurement setup.

This article aims at representing arbitrary known phase differences in terms of additional goals for the minimization problem. Phase differences can be obtained from magnitude only measurements of certain linear combinations as in Costanzo et al. (2005) or by the assumption that a global reference phase will be stable for at least two successive measurements. Finding a global minimum to a cost functional involving both, magnitudes and phase differences, is equivalent to determining the near field up to a global phase shift. By introducing the phase knowledge in terms of minimization goals and not setting phase differences to a fixed value, we allow for solutions which give an overall best approximation for the phase differences and the magnitudes, thus being less prone to measurement errors.

The article is structured as follows. Section 2 briefly revisits the phaseless NFFFT presented in Schnattinger et al. (2014), which is similar to the analysis of the Wirtinger Flow minimizations in Candes et al. (2015) and Zhang and Liang (2016). In Sect. 3, two extensions for the cost functional are presented which introduce phase knowledge to the minimization problem. These implementations are formally analyzed in Sect. 4 for their behavior. Finally Sect. 5 shows numerical evidence for the effectiveness of the proposed methods.

\section{Formulation of the phaseless field transformation}

The task of an NFFFT is to determine the electromagnetic FFs of an antenna from a number of NF measurements. It can be solved as an inverse problem by finding equivalent sources in the AUT volume or on the surface of the AUT volume. The equivalent sources are chosen such that they reproduce the measured NF values. The FF is thereafter easily obtained from the found equivalent currents. Starting from a discrete set of basis functions for the sources (e.g. RWG basis functions in Rao et al., 1982), one obtains a linear equation system

$b=A z$,

where $\boldsymbol{b} \in \mathbb{C}^{M}$ is the vector of $M$ complex NF measurements, $z \in \mathbb{C}^{N}$ contains the $N$ coefficients for the equivalent source basis functions and $\boldsymbol{A} \in \mathbb{C}^{M \times N}$ is the system matrix in which the entry $A_{m n}$ describes the influence of the $n$th basis function on the $m$ th measurement. The minimum mean square error solution of the normal equation system

$z=A^{\dagger} b$

with the pseudo-inverse $\boldsymbol{A}^{\dagger}$ yields the coefficients for the discrete set of equivalent source basis functions for the computation of the corresponding FF. If the dimensions are high, it becomes computationally expensive to calculate the system matrix $\boldsymbol{A}$ explicitly. Then, iterative methods can be utilized to determine the solution of Eq. (2), which only require the evaluation of the matrix vector products $\boldsymbol{A x}$ and $\boldsymbol{A}^{\mathrm{H}} \boldsymbol{x}^{\prime}$, where $\boldsymbol{A}^{\mathrm{H}}$ denotes the Hermitian transpose of $\boldsymbol{A}$. In this work the matrix vector products are evaluated efficiently with the Fast Irregular Antenna Field Transformation Algorithm (FIAFTA) described in Eibert et al. (2015), Schmidt et al. (2008) and Eibert and Schmidt (2009). Due to the hierarchical field representation, the matrix vector products can be evaluated with a computational complexity of $\mathcal{O}(N \log N)$.

By combining the phase retrieval algorithm with FIAFTA, the phaseless NFFFT described here inherits all the positive properties of FIAFTA such as the possibility to work with arbitrary irregular and regular measurement grids, full probe correction for arbitrary probes, and a very flexible source representation with modal field expansions as well as magnetic and/or electric surface currents on a triangular mesh representing the geometry of the AUT.

When the phases of the measurements in $\boldsymbol{b}$ are unknown, we want to find a solution of

$|\boldsymbol{b}|=|\boldsymbol{A} \boldsymbol{z}|$,

or equivalently

$|\boldsymbol{b}|^{\circ 2}=|\boldsymbol{A} \boldsymbol{z}|^{\circ 2}$,

where $|\cdot|$ denotes the elementwise absolute value operator and the exponent $(\cdot)^{\circ 2}$ denotes that the power of 2 is applied on each element of the vector. Since Eq. (4) is a non linear equation, we try to solve the corresponding minimization problem

$\left.\min _{\boldsymbol{z} \in \mathbb{C}^{N}}\left\||\boldsymbol{b}|^{\circ 2}-\right\| \boldsymbol{A} \boldsymbol{z}\right|^{\circ 2} \|_{2}^{2}$

or equivalently

$\min _{\boldsymbol{z} \in \mathbb{C}^{N}}\|\underbrace{\boldsymbol{b}^{*} \circ \boldsymbol{b}}_{\boldsymbol{\beta}}-(\boldsymbol{A} \boldsymbol{z})^{*} \circ(\boldsymbol{A} \boldsymbol{z})\|_{2}^{2}$,

where $\circ$ denotes the Hadamard product, $\boldsymbol{\beta}=\boldsymbol{b}^{*} \circ \boldsymbol{b}$ is the vector of the squared magnitudes of the elements of $\boldsymbol{b}$ and $(\boldsymbol{A} \boldsymbol{z})^{*} \circ(\boldsymbol{A} \boldsymbol{z})$ yields the squared magnitudes of $\boldsymbol{A} \boldsymbol{z}$. For the rest of this paper we will call $\boldsymbol{y}=\boldsymbol{A} \boldsymbol{z}$ virtual measurements and $\beta$ the goal vector. The cost functional $f=$ $\left|\boldsymbol{\beta}-(\boldsymbol{A} \boldsymbol{z})^{*} \circ(\boldsymbol{A} \boldsymbol{z})\right|_{2}^{2}$ in Eq. (6) can be identified as a squared sum of individual differences between the virtual measurements $\boldsymbol{y}=\boldsymbol{A} \boldsymbol{z}$ and the elements of the goal vector $\boldsymbol{\beta}$.

\section{Extension for phase differences}

The main idea of this work is to extend the goal vector $\boldsymbol{\beta}$ by values, which bring information about phase differences 
into the problem. In other words, given we know the phase difference $\phi_{i j}=\phi_{j}-\phi_{i}$ between the measurements $b_{i}$ and $b_{j}$, how can we define a cost functional, which yields the correct phase differences, after its minimization?

\subsection{Magnitude of linear combinations of two measurements}

The (squared) magnitude of a linear combination of the two complex numbers $b_{i}$ and $b_{j}$ carries information about the phase difference between these two numbers as can be seen from

$\left|b_{i}+b_{j}\right|^{2}=\left|b_{i}\right|^{2}+\left|b_{j}\right|^{2}+2\left|b_{i}\right|\left|b_{j}\right| \cos \left(\phi_{i j}\right)$.

The phase difference $\phi_{i j}=\phi_{j}-\phi_{i}$ between the complex measurements $b_{i}$ and $b_{j}$ can uniquely be determined from four magnitudes, namely $\left|b_{i}\right|,\left|b_{j}\right| t,\left|b_{i}+b_{j}\right|$ and $\left|b_{i}+j b_{j}\right|$ for example as in Costanzo and Di Massa (2001). These four linear combinations are not the only possibility to specify the phase differences in terms of magnitudes. Almost any tuple of magnitudes of four different linear combinations of the complex numbers $b_{i}$ and $b_{j}$ will define the phase difference between these two numbers uniquely. The rare tuples which are not suitable to reconstruct the phases can easily be avoided by a careful choice of the linear combinations to be measured. A natural choice for an additional row inside the norm of the cost functional is to use the magnitude of a linear combination of two already existing measurements. The goal value $\beta_{N+1}^{\prime}$ for this linear combination magnitude can be obtained from additional measurements with special probes as in Costanzo et al. (2005) or can easily be computed from known magnitudes and phase differences analogous to Eq. (7). Formally, the cost functional in Eq. (6) can be extended for the newly introduced information by

$$
\begin{aligned}
f^{\prime} & =f+\left|\beta_{N+1}-\right| y_{i}+\left.\left.y_{j}\right|^{2}\right|^{2} \\
& =f+\left|\beta_{N+1}-\right|[\boldsymbol{A} \boldsymbol{z}]_{i}+\left.\left.[\boldsymbol{A} \boldsymbol{z}]_{j}\right|^{2}\right|^{2},
\end{aligned}
$$

with the new goal

$\beta_{N+1}=\left|b_{i}+b_{j}\right|^{2}$.

The additional term introduces an additional penalty for any deviations in the magnitudes of the considered linear combination which in turn can be interpreted as an additional phase constraint according to Eq. (7).

In general, any linear combination of arbitrary measurements can be attached, by extending the cost functional in a similar manner as before. One obtains

$f^{\prime}=f+\left|\beta_{N+1}-\right| \alpha_{1} y_{i}+\left.\left.\alpha_{2} y_{j}\right|^{2}\right|^{2}$

with the new goal

$\beta_{N+1}=\left|\alpha_{1} b_{i}+\alpha_{2} b_{j}\right|^{2}$.
Also an arbitrary number of additional linear combinations can be considered by simply adding more terms like Eq. (10).

Due to the flexibility of the formulation in this chapter, in principle magnitudes of any probe or linear combination of probes can be incorporated into the minimization, without the need of determining phase differences explicitly. However, if the phase differences are known, the magnitudes of any linear combination can be calculated and incorporated as presented.

\subsection{Complex conjugated multiplication}

The phase of a product of a complex number $b_{i}$ and the complex conjugate of another complex number $b_{j}^{*}$ is equal to the phase difference $\phi_{j i}=\phi_{i}-\phi_{j}$ between them:

$b_{i} b_{j}^{*}=\left|b_{i}\right|\left|b_{j}\right| e^{j \phi_{j i}}$.

If the phase difference between two measurements is known, this information can be incorporated into Eq. (6) with usage of this multiplication identity. Consider the extended cost functional

$$
\begin{aligned}
f^{\prime} & =f+\left|\beta_{N+1}-y_{i} y_{j}^{*}\right|^{2} \\
& =f+\left|\beta_{N+1}-[\boldsymbol{A} \boldsymbol{z}]_{i}[\boldsymbol{A} \boldsymbol{z}]_{j}^{*}\right|^{2},
\end{aligned}
$$

with the new goal

$\beta_{N+1}=b_{i} b_{j}^{*}$.

The newly introduced additional term in the cost functional adds a penalty for any deviation of the product $y_{i} y_{j}^{*}$ from its goal and can be interpreted as an additional phase constraint according to Eq. (12).

Similar to the linear combinations it is possible, to extend the cost functional for the complex conjugated multiplication by more than one term, by simply attaching more terms in the presented scheme. It is also possible, to combine linear combinations and complex conjugated multiplication costs in a single cost functional by attaching both of the corresponding cost functional types.

Notice that the extended goal vector $\boldsymbol{\beta}^{\prime}$ contains complex values for the complex conjugated multiplication terms as opposed to real and positive numbers only in the case of magnitudes. This makes the evaluation of the Jacobian, which is needed for many minimization procedures, more difficult.

\section{Analysis of the extended cost functionals}

In this section the behavior of the newly introduced cost functionals will be analyzed. To this end, consider the four cost functionals

$f_{1}=\left|\beta_{1}-\right| y_{i}+\left.\left.y_{j}\right|^{2}\right|^{2}$, 
$f_{2}=\left|\beta_{2}-\right| y_{i}-\left.\left.j y_{j}\right|^{2}\right|^{2}$,

$f_{3}=f_{1}+f_{2}$,

$f_{4}=\left|\beta_{4}-y_{i} y_{j}^{*}\right|^{2}$,

with the goal terms

$\beta_{1}=\left|b_{i}+b_{j}\right|^{2}$,

$\beta_{2}=\left|b_{i}-j b_{j}\right|^{2}$,

$\beta_{4}=b_{i} b_{j}^{*}$.

The variables $y_{i}$ and $y_{j}$ denote the $i$ th and $j$ th virtual measurement and $b_{i}$ and $b_{j}$ for the corresponding (hypothetical) true complex measurements. In general, the virtual measurements $y_{i}$ deviate from the goals $b_{i}$ in magnitude and phase, i.e.

$y_{i}=c_{i} b_{i} e^{j \Delta \phi_{i}}$,

with $c_{i} \in \mathbb{R}^{+}$is the magnitude factor by which the virtual measurement $y_{i}$ deviates from the goal $b_{i}$ and $\Delta \phi_{i} \in[0,2 \pi]$ is the phase difference between $b_{i}$ and $y_{i}$, i.e. $\angle y_{i}=\angle b_{i}+$ $\Delta \phi_{i}$. As discussed previously, only the magnitudes of $b_{i}$ and $b_{j}$ and the linear combinations or the phase difference between them might be known (i.e. the absolute phase of $b_{i}$ and $b_{j}$ is unknown). Since only the phase difference between the virtual measurements $y_{i}$ and $y_{j}$ is relevant, we can identify the term

$\phi_{\epsilon}=\Delta \phi_{i}-\Delta \phi_{j}$

as the error term in the phase difference between $y_{1}$ and $y_{2}$. Remember that $\phi_{j i}=\phi_{i}-\phi_{j}$ is the phase difference between the goals $b_{i}$ and $b_{j}$, while $\Delta \phi_{i}$ and $\Delta \phi_{j}$ are the phase deviations of the virtual measurements from the goals $b_{i}$ and $b_{j}$ respectively, i.e. if the virtual measurements have the same global phase shift to the actual measurements $b_{i}$ and $b_{j}$ (i.e. $\Delta \phi_{i}=\Delta \phi_{j}$ ), the error term $\phi_{\epsilon}$ for the phase difference is zero. The cost functionals $f_{1}$ and $f_{2}$ correspond to linear combinations considered in Sect. 3.1 and the cost functional $f_{3}$ is the sum of the cost functionals $f_{1}$ and $f_{2}$. The cost functional $f_{4}$ corresponds to a complex conjugated multiplication presented in Sect. 3.2.

Figure 1 shows the values of $f_{1}$ to $f_{4}$ dependent on the phase deviation $\phi_{\epsilon}=\Delta \phi_{i}-\Delta \phi_{j}$ in case that the virtual measurements $y_{i}$ and $y_{j}$ have the same magnitudes as th correspondent goals $b_{i}$ and $b_{j}$. The values for $b_{i}=e^{j 55^{\circ}}$ and $b_{j}=2.3 e^{j 0^{\circ}}$ have been chosen arbitrarily to yield cost functionals which plainly show the different behaviors. It can be seen that all four cost functionals have a minimum for $\phi_{\epsilon}=0^{\circ}$. However, the cost functionals $f_{1}$ and $f_{2}$ also show a false minimum for another $\phi_{\epsilon} \neq 0^{\circ}$. For the case of no magnitude deviations, i.e. $c_{i}=c_{j}=1$, the cost functional $f_{3}$ is a scaled version of the cost functional $f_{4}$.

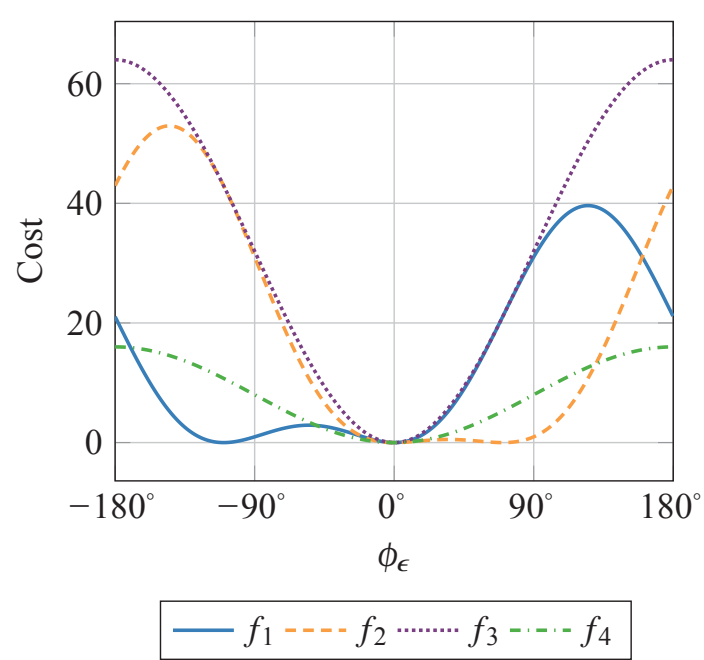

Figure 1. Cost functionals for phase error only. For this example the goal values have been chosen to be $b_{i}=e^{j 55^{\circ}}$ and $b_{j}=2.3 e^{j 0^{\circ}}$.

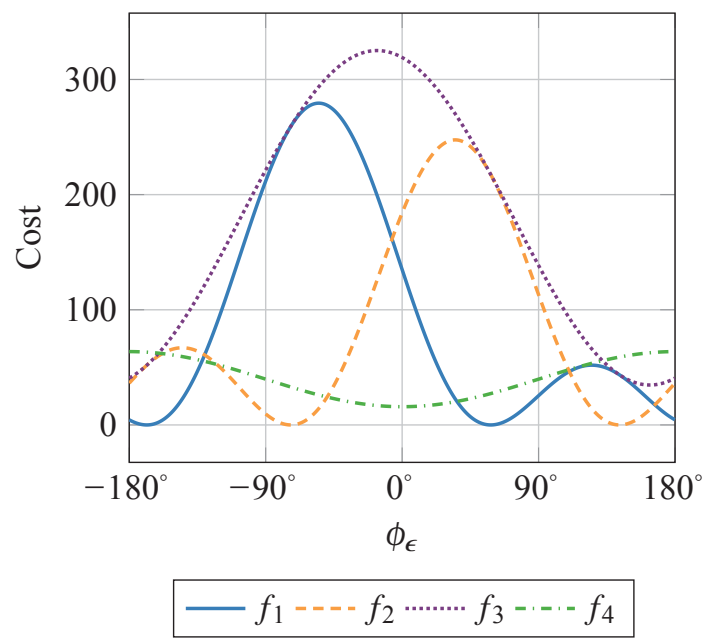

Figure 2. Cost functionals with phase and magnitude error. For this example, additionally to the phase deviation, a multiplicative amplitude deviation of $c_{i}=2.3$ and $c_{j}=1.3$ is assumed.

In the minimization process, while the minimization has not terminated yet, in general there will be phase deviations $\phi_{\epsilon} \neq 0$ as well as magnitude deviations $c_{i} \neq c_{j} \neq 1$. Figure 2 shows the values of the cost functionals dependent on the phase deviation $\phi_{\epsilon}$ for virtual measurements which deviate from the goals in phase as well as in magnitude, viz. $c_{i}=2.3$ and $c_{j}=1.3$. The false minima for $f_{1}$ and $f_{2}$ are more distinct than in Fig. 1 and the cost functionals $f_{1}$ and $f_{2}$ can return zero and thus reach their global minimum even though neither magnitudes nor the phase difference matches the goals. The cost functionals $f_{3}$ and $f_{4}$ have a minimum for a single value of $\phi_{\epsilon}$ only, i.e. they do not suffer from false minima. 
For an analysis, the cost functionals $f_{1}$ to $f_{4}$ can be expressed in terms of their magnitude and phase deviation. For the cost functional $f_{1}$ we have

$$
\begin{aligned}
f_{1} & =\left|\beta_{1}-\right| y_{i}+\left.\left.y_{j}\right|^{2}\right|^{2} \\
& =|| b_{i}+\left.b_{j}\right|^{2}-\left.\left|c_{i} b_{i} e^{j \Delta \phi_{i}}+c_{j} b_{j} e^{j \Delta \phi_{j}}\right|^{2}\right|^{2} \\
& =\left.|| b_{i}\right|^{2}\left(1-c_{i}^{2}\right)+\left|b_{j}\right|^{2}\left(1-c_{j}^{2}\right) \\
& +\left.2\left|b_{i}\right|\left|b_{j}\right| \operatorname{Re}\left\{e^{j \phi_{j i}}-c_{1} c_{2} e^{j\left(\phi_{j i}+\Delta \phi_{i}-\Delta \phi_{j}\right)}\right\}\right|^{2} \\
& =\left.|| b_{i}\right|^{2}\left(1-c_{i}^{2}\right)+\left|b_{j}\right|^{2}\left(1-c_{j}^{2}\right) \\
& +\left.2\left|b_{i}\right|\left|b_{j}\right| \operatorname{Re}\left\{e^{j \phi_{j i}}-c_{1} c_{2} e^{j\left(\phi_{j i}+\Phi_{\epsilon}\right)}\right\}\right|^{2} .
\end{aligned}
$$

Accordingly, for the cost functional $f_{2}$ we have

$$
\begin{aligned}
f_{2} & =\left|\beta_{2}-\right| y_{i}-\left.\left.j y_{j}\right|^{2}\right|^{2} \\
& =|| b_{i}+\left.j b_{j}\right|^{2}-\left.\left|c_{i} b_{i} e^{j \Delta \phi_{i}}-j c_{j} b_{j} e^{j \Delta \phi_{j}}\right|^{2}\right|^{2} \\
& =\left.|| b_{i}\right|^{2}\left(1-c_{i}^{2}\right)+\left|b_{j}\right|^{2}\left(1-c_{j}^{2}\right) \\
& +\left.2\left|b_{i}\right|\left|b_{j}\right| \operatorname{Im}\left\{e^{j \phi_{j i}}-c_{1} c_{2} e^{j\left(\phi_{j i}+\phi_{\epsilon}\right)}\right\}\right|^{2} .
\end{aligned}
$$

The cost functionals $f_{1}$ and $f_{2}$ deviate from each other only by evaluating the real and imaginary part of $e^{j \phi_{j i}}-$ $c_{i} c_{j} e^{j\left(\phi_{j i}+\phi_{\epsilon}\right)}$ respectively. Thus, they suffer from the same kind of undesired behavior. For any given magnitude deviation $c_{i}$ and $c_{j}$, both cost functionals have two minima for two different values of $\phi_{\epsilon}$ (there are two numbers on the unit circle which have the same imaginary or real part respectively). The desired behavior would be that the cost functionals have a single minimum for $\phi_{\epsilon}=0$ only. The occurring false minimum reflects the fact that a single linear combination magnitude does not uniquely define the phase difference between the two complex numbers $b_{i}$ and $b_{j}$. Note, that two distinct minima might occur for each of the cost functionals $f_{1}$ and $f_{2}$ even, when the magnitudes $\left|y_{i}\right|=\left|b_{i}\right|$ and $\left|y_{j}\right|=\left|b_{j}\right|$ are equal to the desired magnitudes, i.e. $c_{1}=c_{2}=1$.

For the analysis of cost functional $f_{3}$, we introduce the auxiliary variable

$A=\left|b_{i}\right|^{2}\left(1-c_{i}^{2}\right)+\left|b_{j}\right|^{2}\left(1-c_{j}^{2}\right)$.

By identifying Eq. (24) as the squared magnitude of the real part and Eq. (25) as the squared magnitude of the imaginary part of the same complex number, $f_{3}$ can be written as

$$
\begin{aligned}
f_{3} & =f_{1}+f_{2} \\
& =|(1+j) A+2| b_{i}|| b_{j}\left|\left(e^{j \phi_{j i}}-c_{1} c_{2} e^{j\left(\phi_{j i}+\phi_{\epsilon}\right)}\right)\right|^{2} \\
& =\left|(1+j) A e^{-j \phi_{j i}}+2\right| b_{i}|| b_{j}\left|\left(1-c_{1} c_{2} e^{j \phi_{\epsilon}}\right)\right|^{2}
\end{aligned}
$$

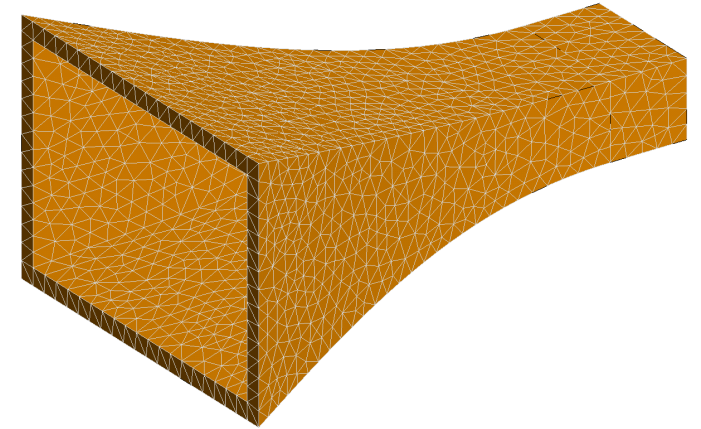

Figure 3. Mesh defining the RWG unknowns for transforming synthetic data of a horn antenna.

$$
=|B-2| b_{i}|| b_{j}\left|c_{i} c_{j} e^{j \phi_{\epsilon}}\right|^{2}
$$

with the auxiliary term $B=(1+j) A e^{-j \phi_{j i}}+2\left|b_{i}\right|\left|b_{j}\right|$. From the last row of Eq. (27) it is clear that the cost functional $f_{3}$ is minimal only for the single value of $\phi_{\epsilon}$, when it equals the phase of $B$ shifted by $180^{\circ}$. The cost functional obtains its minimum for $c_{i}=c_{j}=1$ and $\phi_{\epsilon}=0$, as intended, however for general magnitude deviations $c_{i} \neq 1$ and $c_{j} \neq 1$, the cost functional $f_{3}$ may obtain its minimum at $\phi_{\epsilon} \neq 0$. Some tuples $\left(c_{i}, c_{j}, \phi_{\epsilon}\right) \neq(1,1,0)$ can also lead to $f_{3}=0$. However, together with the minimization of the magnitudes as described in Sect. 2, the cost functional has a unique global minimum (up to a global phase) for $y_{i}$ and $y_{j}$.

The cost functional $f_{4}$ can be rewritten in the form

$$
\begin{aligned}
f_{4} & =\left|\beta_{4}-y_{i} y_{j}^{*}\right|^{2} \\
& =\left|b_{i} b_{j}^{*}-b_{i} b_{j}^{*} c_{i} c_{j} e^{j \phi_{\epsilon}}\right|^{2} \\
& =\left|b_{i}\right|^{2}\left|b_{j}\right|^{2}\left|1-c_{i} c_{j} e^{j \phi_{\epsilon}}\right|^{2} .
\end{aligned}
$$

The cost functional $f_{4}$ has a global minimum for $c_{i}=c_{j}=1$ and $\phi_{\epsilon}=0$. Also independent on the deviation between $y_{i}$ and $b_{i}$ and $y_{j}$ and $b_{j}$, the minimum for $f_{3}$ occurs a $\phi_{\epsilon}=0$. However the cost functional $f_{3}$ can return zero also for any combination of magnitude deviations $c_{i}=\left(1 / c_{j}\right) \neq 1$. Thus for a unique global minimum with $c_{i}=c_{j}=1$ and $\phi_{\epsilon}=0$ the cost functional $f_{3}$ has to be minimized together with the magnitude cost functional from Sect. 2.

\section{Numerical results}

The effectiveness of the presented methods is shown by numerical examples. The NF data has been generated synthetically according to Schmidt et al. (2011) at $3 \mathrm{GHz}$. The behavior of a horn antenna has been simulated by 2232 dipoles arranged on a surface enclosing a CAD model of a real horn antenna depicted in Fig. 3. The horn antenna model aperture 


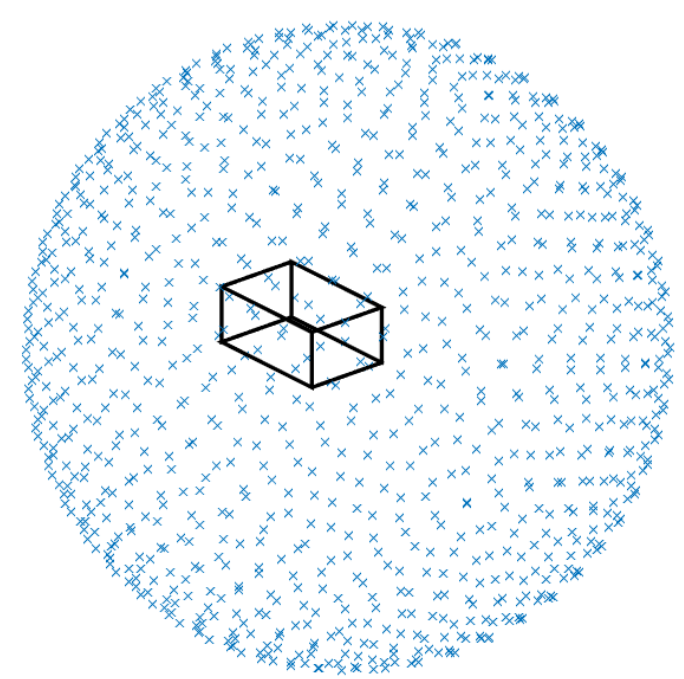

Figure 4. Location of the sample points.

is $227.40 \mathrm{~mm} \times 151.60 \mathrm{~mm}$ in size. The other side of the antenna builds a transition to a rectangular waveguide of dimensions $72.14 \mathrm{~mm} \times 34.04 \mathrm{~mm}$. The horn length is $350 \mathrm{~mm}$ and the taper is a $(1-\cos )$-taper. The excitations for the dipoles have been obtained approximately from a simulation in CST MICROWAVE STUDIO 2016 (CST, 2016). For the NFFFT the triangles on the same mesh has been used for RWG basis functions (Rao et al., 1982), i.e. the vector $z$ in Eq. (1) contains the coefficients of each RWG basis function.

As measurement probes serve Hertzian dipoles. Two probes at a time form a measurement pair which will be evaluated for the phase differences. A pair consists of two, horizontally separated dipoles spaced two wavelengths apart. The measurement points, i.e. the centers of the dipole pairs, are located on spirals on a spherical surface around the AUT. Figure 4 shows the 1000 locations of the pair centers along with a black cuboid denoting the location of the AUT. The spiral sampling shown in Fig. 4 is more uniformly distributed than the usual spherical sampling which has equidistant angular steps in $\vartheta$ and $\varphi$ direction. Since in general two independent polarizations are needed in NF measurements, the same measurements have been obtained with rotated dipoles. Thus we have 4000 measurements (not counting any linear combinations) since at each measurement location two measurements - one to the left and one to the right - have been obtained with two polarizations. Any linear combination and phase differences can be obtained from the complex valued synthetic dipole outputs. In the following linear combinations and phase differences have been considered only for corresponding dipole pairs. Note, that the locations of dipoles of neighboring pairs do not interfere in general, which means that the absolute phases of the measurements at the individual dipoles cannot be determined by iterating through a chain of known phase differences.
Table 1. Minimization overview.

\begin{tabular}{lrrrr}
\hline Cost & $\begin{array}{r}\text { No. of } \\
\text { iterations }\end{array}$ & $\varnothing$ FF error & $\begin{array}{r}\text { max } \\
\text { FF error }\end{array}$ & Time \\
\hline$f$ & 1285 & $-32.4 \mathrm{~dB}$ & $-14.9 \mathrm{~dB}$ & $1.20 \mathrm{~min}$ \\
$f+f_{1}$ & 1075 & $-36.4 \mathrm{~dB}$ & $-14.3 \mathrm{~dB}$ & $1.14 \mathrm{~min}$ \\
$f+f_{3}$ & 12960 & $-81.9 \mathrm{~dB}$ & $-54.4 \mathrm{~dB}$ & $10.92 \mathrm{~min}$ \\
$f+f_{4}$ & 9493 & $-85.7 \mathrm{~dB}$ & $-62.2 \mathrm{~dB}$ & $6.66 \mathrm{~min}$ \\
\hline
\end{tabular}

Four scenarios have been considered. In the first scenario, the coefficients in $z$ are retrieved from magnitude only measurements as in Sect. 2. In the second scenario, additionally to the magnitudes of the single dipole outputs, the magnitudes of the sum of the outputs of each dipole pair have been considered according to Sect. 3.1 and Eq. (24). In the third scenario also the magnitudes of the phase shifted sums have been considered as in Eq. (27). Finally, in the fourth scenario the phase knowledge has been included in terms of conjugated multiplication as described in Sect. 3.2 and in Eq. (28). For all scenarios, the corresponding cost functional has been minimized with a L-BFGS procedure (Nocedal and Wright, 2006), a memory limited version of the Broyden-FletcherGoldfarb-Shanno algorithm (named after its inventors) in the family of quasi-Newton methods (Nocedal and Wright, 2006). The algorithm terminates, once an insufficient relative decrease in the cost functional is observed. The initial conditions have been chosen to be $z_{i}=1+1 j \forall i$ for each basis function in all scenarios. After the minimization has finished, the FF is calculated from the retrieved sources in $z$. Table 1 shows an overview over the four scenarios. The different timings are mostly due to the different number of iterations. All minimizations used roughly the same memory of about $450 \mathrm{MB}$. For Table 1, the complete FF has been considered, not only the cuts which are shown in the following subsections.

\subsection{Magnitude only measurements via cost functional $f$}

The dashed blue line in Fig. 5 shows the cut of the copolar E-field component in the E-plane of the retrieved FF from magnitude only NFFFT. The orange solid line denotes the ideal FF, which has been computed from the original source dipoles. Both, the reference and the retrieved FF, are normalized to their maximum respectively. The dotted purple line shows the difference between both curves. The maximal error in the FF (relative to the maximum of any FF) is larger than $-20 \mathrm{~dB}$. Figure 6 shows the magnitude of the cost functional at each iteration, normalized to its initial value. The termination criterion is met after 1285 iterations and the progression of the curve hints to the cost functional being stuck in a local stationary point. Especially outside the main lobe, the retrieved FF pattern does not match the reference. As 


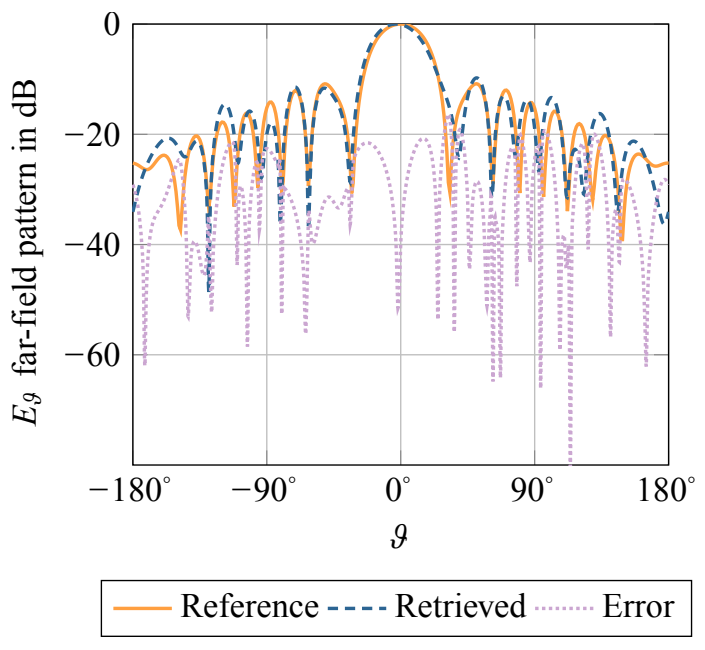

Figure 5. Retrieved FF from magnitude only data, $\varphi=90^{\circ}$.

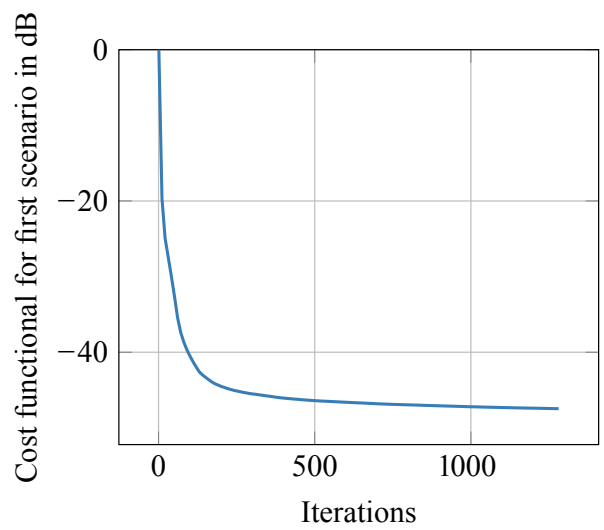

Figure 6. Progress of the minimization of the cost functional for magnitude only data.

shown in Table 1 , the maximum FF error is at $-14.9 \mathrm{~dB}$ and the average FF error is $-32.4 \mathrm{~dB}$.

\subsection{Magnitudes of single measurements and of pair sums via cost functional $f+f_{1}$}

For the second scenario, also the magnitudes of the complex sum of the outputs of each dipole pair have been considered. The E-plane cut of the retrieved copolar FF component can be found in Fig. 7. The values in Table 1 suggest that there hardly is any improvement compared to the magnitude only minimization. Even though the retrieved FF in Fig. 7 seems to show a better result than Fig. 5, especially outside the main lobe, the error level is still very high and the minimization again seems to be captured at a local stationary point which is different from the one in the magnitude only minimization, even though the initial guess has been the same. This agrees with the analysis from Sect. 4, in which it was stated that false minima may occur for the additional cost functional parts in $f_{1}$. Nevertheless, the usage of the magnitude of a

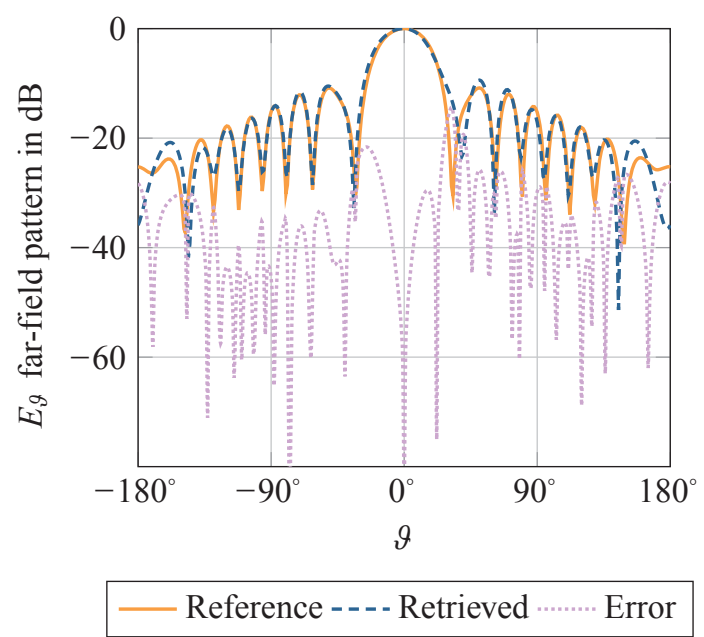

Figure 7. Retrieved FF from magnitudes and one linear combination data, $\varphi=90^{\circ}$.

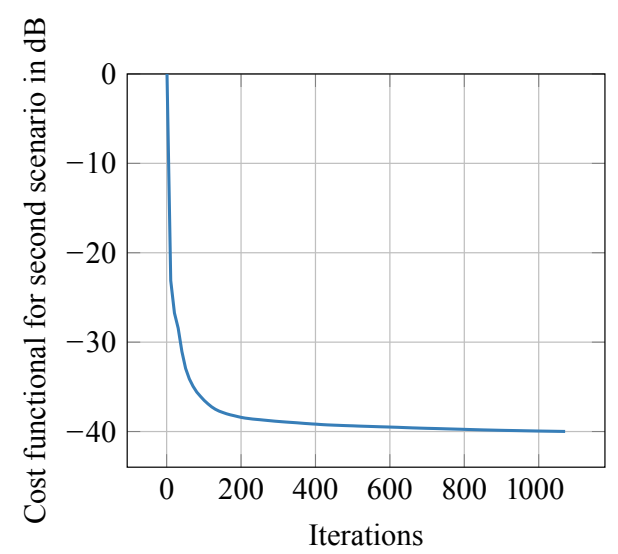

Figure 8. Progress of the minimization of the cost functional for magnitude data combined with one linear combination magnitude per diploe pair.

linear combination has some effect since it leads to a termination at a different stationary point than the magnitude only minimization. In some cases this may lead to a situation in which local stationary points from the magnitude only data are avoided. In this particular case, the minimization does not progress anymore after 1075 iterations, as shown in Fig. 8.

\subsection{Magnitudes of single measurements, of pair sums, and of phase shifted pair sums via cost functional $f+f_{3}$}

In the third scenario additionally to the magnitude only minimization objectives also the two (squared) magnitudes of the linear combinations $\left|b_{i}+b_{j}\right|^{2}$ and $\left|b_{i}-j b_{j}\right|^{2}$ have been considered for the dipole pairs. Figure 9 shows the retrieved copolar FF in the E-plane. With a maximum FF error of $-54 \mathrm{~dB}$ it can be concluded that the minimization process 


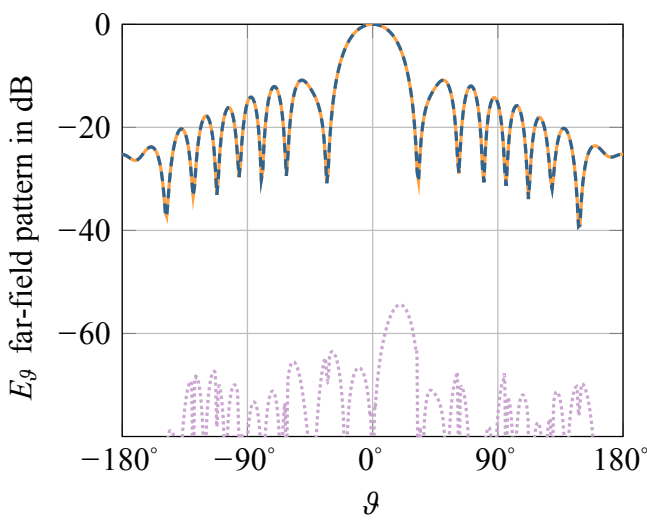

- Reference ---- Retrieved …….. Error

Figure 9. Retrieved FF from magnitudes and two linear combinations data, $\varphi=90^{\circ}$.

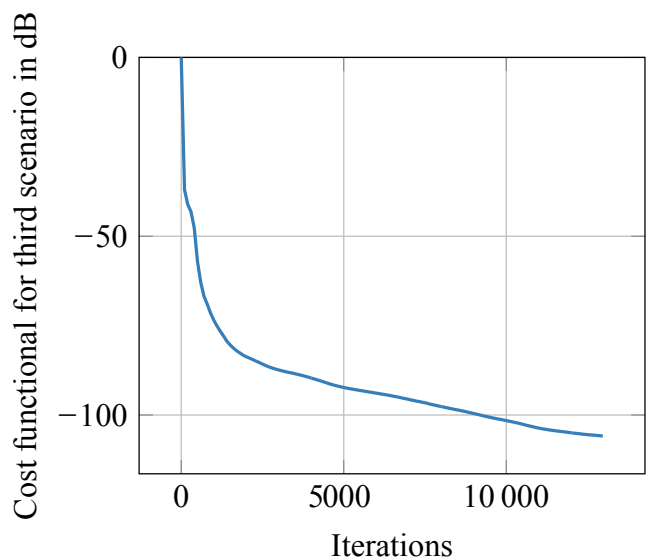

Figure 10. Progress of the minimization of the cost functional for magnitude data combined with two orthogonal linear combination magnitudes per dipole pair.

reached the minimum which corresponds to the correct near fields. This observations supports the conclusions of Sect. 4. The progression of the cost functional shown in Fig. 10 also shows that the minimization did not reach its minimal value before at least ten times the number of iterations of the first two scenarios but also shows a steady decrease. This suggests that all local stationary points have been avoided thanks to the additional objectives for the linear combinations.

\subsection{Magnitudes of single measurements and conjugated multiplication between pairs via cost functional $f+f_{4}$}

In the fourth scenario, parallel to the magnitude only goals, the conjugated multiplications described in Sect. 3.2 have been minimized for the dipole pairs. Figure 11 shows the retrieved copolar FF pattern in the E-plane. Similar to the previous scenario, the retrieved FF coincides with the ref-

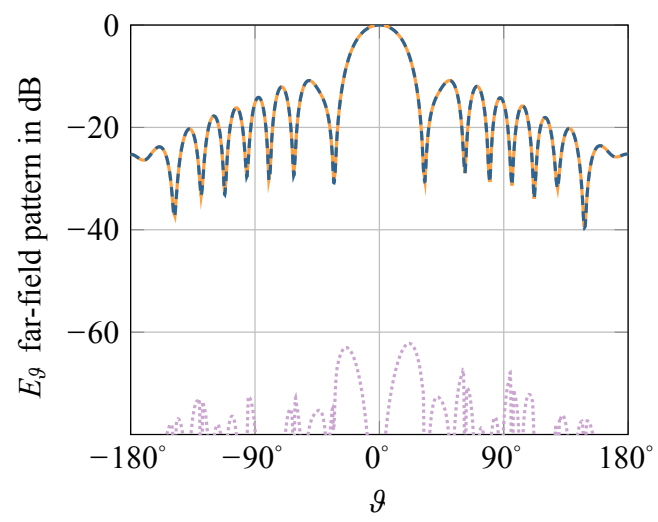

—- Reference ---- Retrieved …...... Error

Figure 11. Retrieved FF from magnitudes and conjugated multiplication data, $\varphi=90^{\circ}$.

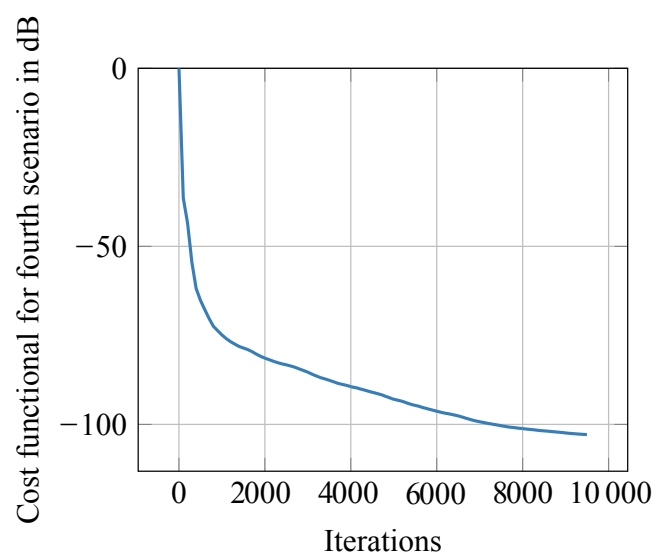

Figure 12. Progress of the minimization of the cost functional for magnitudes combined with conjugated multiplication data per dipole pair.

erence. As stated in Table 1, the maximum FF error is less than $-60 \mathrm{~dB}$. The retrieved FF as well as the progression in Fig. 12 suggest that any local stationary points have been avoided and the correct NF has been retrieved.

\section{Conclusions}

The phaseless near-field (NF) far-field (FF) transformation (NFFFT) can be extended to incorporate phase knowledge in terms of phase differences between measurements and magnitudes of linear combinations. The cost functional for the non linear minimization is formally extended by additional terms which penalize the difference between the virtual measurements and the correspondent goals. The exact build-up of these goals introduces the phase knowledge either in terms of complex conjugated multiplication or in terms of linear combinations. It has been shown that both new cost functionals 
behave differently during the minimization procedure. When the phase goals are chosen with care, the cost functional exhibits a unique global minimum (up to a global phase) which corresponds to the fully restored radiated NF. From this restored NF, the FF can be computed easily with means of standard NFFFT. For the cost functionals with a global minimum corresponding to the correct NF, the FF has been retrieved with a maximum error of up to $-62 \mathrm{~dB}$. However, even though an unique global minimum exists for the radiated fields, the minimization may not converge to this minimum due to the non linearity of the problem. In such a case, the minimization runs into a local stationary point. Incorporating phase knowledge can help to avoid stationary points in this case. This way, one can retrieve a barely distorted FF without the need of full phase measurements.

Data availability. Underlaying data is available from the corresponding author upon request.

Competing interests. The authors declare that they have no conflict of interest.

Disclaimer. The responsibility for the content of this publication is with the authors.

Acknowledgements. This work has been supported by the German Federal Ministry for Economic Affairs and Energy under Contract No. 50YB1512.

This work was supported by the German Research

Foundation (DFG) and the Technische Universität

München within the funding programme

Open Access Publishing.

Edited by: R. Schuhmann

Reviewed by: two anonymous referees

\section{References}

Bauschke, H. H., Combettes, P. L., and Luke, D. R.: Phase retrieval, error reduction algorithm, and Fienup variants: a view from convex optimization, J. Opt. Soc. Am. A, 19, 1334-1345, 2002.

Candes, E. J., Strohmer, T., and Voroninski, V.: Phaselift: Exact and stable signal recovery from magnitude measurements via convex programming, Commun. Pur. Appl. Math., 66, 1241-1274, 2013.

Candes, E. J., Li, X., and Soltanolkotabi, M.: Phase retrieval via Wirtinger flow: Theory and algorithms, IEEE T. Inform. Theory, 61, 1985-2007, 2015.

Costanzo, S. and Di Massa, G.: An integrated probe for phaseless plane-polar near-field measurements, Microw. Opt. Techn. Let., 30, 293-295, 2001.
Costanzo, S., Di Massa, G., and Migliore, M. D.: A novel hybrid approach for far-field characterization from near-field amplitudeonly measurements on arbitrary scanning surfaces, IEEE T. Antenn. Propag., 53, 1866-1874, 2005.

CST: CST MICROWAVE STUDIO 2016, available at: https://www. cst.com/products/cstmws (last access: 28 March 2017), 2016.

Eibert, T. F. and Schmidt, C. H.: Multilevel fast multipole accelerated inverse equivalent current method employing Rao-WiltonGlisson discretization of electric and magnetic surface currents, IEEE T. Antenn. Propag., 57, 1178-1185, 2009.

Eibert, T. F., Kilic, E., Lopez, C., Mauermayer, R. A., Neitz, O., and Schnattinger, G.: Electromagnetic Field Transformations for Measurements and Simulations (Invited Paper), Prog. Electromagn. Res., 151, 127-150, 2015.

Fienup, J. R.: Phase retrieval algorithms: A comparison, Appl. Optics, 21, 2758-2769, 1982.

Gerchberg, R. W.: A practical algorithm for the determination of phase from image and diffraction plane pictures, Optik, 35, 237246, 1972.

Netrapalli, P., Jain, P., and Sanghavi, S.: Phase retrieval using alternating minimization, Adv. Neur. In., 2796-2804, 2013.

Nocedal, J. and Wright, S.: Numerical optimization, 2nd Edn., Springer Science \& Business Media, 2006.

Rao, S., Wilton, D., and Glisson, A.: Electromagnetic scattering by surfaces of arbitrary shape, IEEE T. Antenn. Propag., 30, 409418, 1982.

Schmidt, C., Schobert, D., and Eibert, T.: Electric dipole based synthetic data generation for probe-corrected near-field antenna measurements, in: Proceedings of the 5th European Conference on Antennas and Propagation (EUCAP), 3269-3273, 2011.

Schmidt, C. H., Leibfritz, M. M., and Eibert, T. F.: Fully probecorrected near-field far-field transformation employing plane wave expansion and diagonal translation operators, IEEE T. Antenn. Propag., 56, 737-746, 2008.

Schnattinger, G., Lopez, C., Kiliç, E., and Eibert, T. F.: Fast near-field far-field transformation for phaseless and irregular antenna measurement data, Adv. Radio Sci., 12, 171-177, https://doi.org/10.5194/ars-12-171-2014, 2014.

Waldspurger, I., d'Aspremont, A., and Mallat, S.: Phase recovery, maxcut and complex semidefinite programming, Math. Program., 149, 47-81, 2015.

Wu, X., Liu, H., and Yan, A.: X-ray phase-attenuation duality and phase retrieval, Opt. Lett., 30, 379-381, 2005.

Yurtsever, A., Hsieh, Y.-P., and Cevher, V.: Scalable convex methods for phase retrieval, in: 2015 IEEE 6th International Workshop on Computational Advances in MultiSensor Adaptive Processing (CAMSAP), IEEE, 381-384, https://doi.org/10.1109/CAMSAP.2015.7383816, 2015.

Zhang, H. and Liang, Y.: Reshaped Wirtinger Flow and Incremental Algorithm for Solving Quadratic System of Equations, arXiv preprint, arXiv:1605.07719, 2016. 